\title{
A literature review of human factors and ergonomics within the pharmacy dispensing process
}

\author{
Natalie M. Weir
}

Rosemary Newham

Marion Bennie

University of Strathclyde 
1 A literature review of human factors and ergonomics within the pharmacy dispensing 2 process

\section{1. Introduction}

4 The discipline of Human Factors - also commonly referred to as Ergonomics - centres on the complexity 5 within work systems, and how the interaction between people and the working environment can 6 influence safety, performance and wellbeing. ${ }^{1,2}$ The term was first described by Jastrzebowski in 1857 7 as 'the study of work' $^{3}$, and applications of Human Factors/Ergonomics (HFE) include physical ergonomics (e.g. the physical activity involved within work systems); cognitive ergonomics (e.g. the mental processes involved in work elements); and organisation ergonomics (e.g. the organisational structures, policies and processes). ${ }^{3}$ Applying the discipline of HFE involves a whole systems approach, ${ }^{4,5}$ which has been defined by the World Health Organisation (WHO) as 'the study of all the factors that make it easier to do the work in the right way'. ${ }^{6}$ It explores human involvement in conjunction with the tools and technology used; tasks performed; the immediate workspace; the wider physical environment; the organisational arrangements and contexts; and the social, political and economic climate. ${ }^{7}$ Common objectives of applying a HFE approach are to increase the safety and reliability of systems; improve the working environment; increase the ease of use and user acceptance of technology; and reduce loss of time, fatigue and physical stress. ${ }^{3}$ Rail systems, ${ }^{5}$ the construction industry, ${ }^{8}$ aviation, ${ }^{9}$ and technological innovations ${ }^{10}$ have each been extensively explored from a HFE perspective.

Within healthcare, adopting a HFE approach is considered valuable due to the inherent complexity of healthcare contexts; multiple stakeholders work within a myriad of dynamic settings including hospitals, general practices, pharmacies and clinics. ${ }^{11}$ Moreover, system failures within healthcare can result in unintended consequences which compromises patient safety, and improved understanding of healthcare systems allow strategies to be developed to detect, correct and mitigate problems ${ }^{12}$ ultimately improving system resilience and ensuring patient safety..$^{13}$ The earliest application of HFE within healthcare have been described in the $1980 \mathrm{~s},{ }^{14}$ and was later pioneered by James Reason in 1995 who emphasised designing safe healthcare systems, embracing a culture of understanding, and the importance of exploring adverse events and their causation. ${ }^{15}$ Since then, the field of HFE has been extensively applied to healthcare setting internationally, with HFE research conducted in the US, the UK, Canada and China, for example. ${ }^{16}$ In 2010, a Human Factors Advisory Board was established to identify how human factors could be embedded within the UK National Health Service (NHS), which emphasised the exploration of 'never events' and recommended that NHS regions be supported to understand HFE. ${ }^{17}$ Within the U.S. health service, HFE has been integral in the development and application of medical technology, with both the Food and Drug Administration (FDA) and the U.S. 
Department of Veterans Affairs considering HFE crucial in developing "human-centred" technology to ensure its safe and effective use. ${ }^{18,19}$

The pharmacy setting is of potential interest within the field of HFE considering the high-risk nature of the work activities and the risk of medical errors. ${ }^{20} \mathrm{~A}$ medical error is defined by the WHO as a preventable, unintended consequence of a medical intervention, which can range from patients receiving the wrong medicinal treatment or surgical procedure, to a misdiagnosis. ${ }^{21}$ Variable system performance within pharmacy settings can result in medical errors which can cause serious harm or death, ${ }^{22}$ with key areas of risk for medical errors being the transcription of prescribed medication and the preparation and selection of pharmaceutical products. ${ }^{22}$ As these areas of risk typically exist throughout the pharmacy dispensing process, these sub-set of medical errors are referred to as dispensing errors, with common examples including the supply of the incorrect drug, strength, dosage form, quantity or instructions to patients. ${ }^{23}$ Although the frequency of such dispensing errors varies considerably internationally and is compounded by the application of varied calculation methods, within the community pharmacy setting in the UK, the incidence of dispensing errors has been reported as $0.04-3.32 \% .^{23}$

Additional to the inherent risk of dispensing, the pharmacy setting is both complex and dynamic, which poses challenges to patient safety. Internationally, the pharmacy setting is experiencing notable role expansion. New services offered by pharmacy staff include the administration of vaccines, ${ }^{24}$ smoking cessation support, ${ }^{25}$ and medication reviews, ${ }^{26,} 27$ and pharmacists have adopted prescribing responsibilities in both acute and secondary care settings. ${ }^{28,}{ }^{29}$ As a result, there have been considerable workforce changes to improve the efficiency and workflow of pharmacy work systems, such as the introduction of pharmacy technicians performing accuracy checks on dispensed medication, and the implementation of novel technologies such as automated dispensing and electronic prescribing (ePrescribing). ${ }^{30,31}$

Considering the high risk, complex and dynamic nature of the pharmacy dispensing process, applying a HFE approach to explore this setting and its transformational change may support the development of safe and efficient work processes. However, an understanding of HFE and safety within pharmacy is limited, ${ }^{11,32}$ and there is a dearth of knowledge on how HFE approaches and principles have been applied to the pharmacy dispensing setting. Considering the current evolution of the pharmacy profession, it is timely to review the present state of HFE research within this setting. Therefore, the aim of this paper is to identify and review studies which have explored the pharmacy dispensing process from a HFE perspective, with the following objectives:

1. Report study characteristics and the methodological approaches adopted 
2. Identify and categorise the focus of the studies

3. Synthesise the results of the studies and the recommendations posed for future research

\section{Method}

\section{Inclusion criteria of studies}

Eligible studies were those which explored the pharmacy dispensing process from a HFE perspective. The dispensing process was considered to involve the following tasks: receiving the prescription; interpreting the prescription; selecting and labelling the prescribed item; final check of the dispensed item; recording action taken; and issuing the prescribed medication with instructions and advice. ${ }^{33}$ Studies were included if they applied a methodology or philosophy derived from HFE (e.g. if they conducted an analysis of the systems existing within the dispensing process), even if it was not explicitly stated that the study applied a "human factors", "ergonomics" or "socio-technical" approach within the manuscript. Studies from any country were eligible for inclusion. Qualitative, quantitative and mixed-method studies were included from peer-reviewed journals, conference proceedings and poster presentations. Books, editorials, lecture commentaries and other sources reporting nonoriginal research were excluded. Studies were excluded if they were conducted outwith the pharmacy dispensary setting (e.g. on medical wards), focused on a pharmacy service which did not involve the dispensing process (e.g. over-the-counter sale of medicines), or centred on pharmaceutical compounding (i.e. the combining, mixing, or altering of pharmaceutical ingredients). Studies conducting quantitative prospective risk assessment of the dispensing process were excluded as this has been reviewed recently by Stojkovic et al in $2017,{ }^{22}$ although the findings of the current review will be discussed in context with that review.

\section{Search strategy}

The databases Medline, EMBASE and PsycINFO were searched on the $27^{\text {th }}$ of November 2018 from their inception. Supplementary File 1 shows the full Medline search strategy. The search was limited to the English language and covered all studies available up until the search date. After the databases were searched, a supplementary snowball search involved screening the reference lists of identified studies and other publications by the studies' authors. For studies which were not accessible, the authors were contacted via email to obtain full texts.

\section{Study selection}

Study titles and abstracts were screened by the primary reviewer NW, with studies of potential relevance progressing onto full text screening. Where there was dubiety over a study's eligibility for inclusion, RN was consulted and through discussion a decision made on whether to include the study. 
All data extraction was conducted by NW and peer reviewed by RN. Data extracted were: study authors, publication date, journal, country, setting (i.e. community or hospital pharmacy), aim/objective(s), methods applied, theory/model/framework applied or developed, key findings, recommendations posed for future research, and conclusions. Descriptive statistics were used to report study characteristics and methodological approaches. An inductive content analysis of the aim/objective(s) of each study was conducted by NW, whereby the aim of each study was read and through an iterative process commonalities between the aims were identified. This process identified three distinct study foci. NW and RN independently applied this categorisation to the included studies, with any difference in categorisation resolved by NW and RN through discussion. Key findings of the studies and recommendations posed for future research were extracted and narrated using a descriptive narrative synthesis method to facilitate the integration of results derived from a variety of methodologies. ${ }^{34-36}$

\section{Results}

\section{Characteristics of studies}

The titles and abstracts of 1127 articles were screened, with 195 progressing onto full text screening (Fig. 1). Of these, 32 studies were eligible for inclusion. ${ }^{37-68}$

[Fig. 1. Flow Chart of included studies to be displayed here]

Data extracted from each study is summarised in Supplementary File 2. Twenty-seven studies were conducted within the community pharmacy setting, ${ }^{38,39,41-45,47,49-67}$ with five conducted within hospital pharmacy settings. ${ }^{37,40,46,48,68}$ The most common county of origin for the studies was the US ( $n=16$, 48.5\%), 10 studies (30.3\%) originated in the UK, with the remainder originating in Australia $(n=1$, $3.0 \%)$, Ghana $(n=1,3.0 \%)$, Greece $(n=1,3.0 \%)$, Norway $(n=1,3.0 \%)$ and Saudi Arabia $(n=1,3.0 \%)$. One study did not disclose its geographical location. ${ }^{56}$ The majority of studies were published from 2010 onwards ( $n=23,71.8 \%)$, and over time an increase in the number of publications centring on HFE within the pharmacy dispensing setting was observed (Fig. 2). Although the focus of all studies were within the wider domain of HFE, 15 studies (46.8\%) did not explicitly refer to a "human factors", "sociotechnical", or "ergonomics" approach within the manuscript. ${ }^{37,39,40,44,46,48-50,54-56,61,65-67}$ The studies were published in various types of journals or presented at different types of conferences including those which were pharmacy focused $(n=14,43.8 \%), 37,38,42,44,46,50-53,55,60,61,66,67$ healthcare-focused $(n=8,25.0 \%),{ }^{41}, 43,49,54,57,59,62,64$ HFE-focused $(n=5,15.6 \%),{ }^{40,45}, 56,65,68$ and safety-focused $(n=5$, $15.6 \%) .{ }^{39,47,48,59,63}$ 
There were 17 different types of data collection methods applied by the studies, and the majority of studies ( $n=23,71.9 \%$ ) applied multiple data collection approaches (Table 1). Twenty-two studies $(68.8 \%)$ used primary data collection methods (i.e. data collected by a researcher from first-hand sources), with qualitative approaches being the most common. Three studies (13.6\%) used secondary data collection methods, whereby the data was already collected for other purposes, and seven studies (21.9\%) used both primary and secondary data collection methods.

[Table 1. Data collection methods applied by the studies $(n=32)$ to be displayed here]

\section{Application of a model, framework or theory}

Ten of the included studies (31.3\%) applied a model, framework or theory within the study design. ${ }^{38,}$ $43,45-47,52,55,57,58,60$ The Sociotechnical systems (STS) theory was applied in two studies conducted by Odukoya and colleagues, ${ }^{57,58}$ with the remaining models, frameworks or theories applied once. This included a range of work systems models, including: the Systems Engineering Initiative for Patient Safety (SEIPS) model, ${ }^{60}$ the Human Factors Framework, ${ }^{38}$ Safety-I and Safety-II theory, ${ }^{47}$ Leavitt's organizational model, ${ }^{55}$ and Reason's accident causation model. ${ }^{46}$ Three studies developed a new model, framework or theory: the Community Health Integration through Pharmacy Process and Ergonomics Redesign (CHIPPER) framework, ${ }^{45}$ a Sociotechnical model which stratifies pharmacies as "technically oriented", "socially oriented" or "improvising", ${ }^{43}$ and a medication safety event conceptual framework. ${ }^{52}$

\section{Analytical approaches}

Qualitative analysis was the most commonly applied analytical approach $(n=17,53.1 \%) .38,41-43,46,47,50$ $52,55,57-60,62,65,67$ This was followed by systems representation analysis ( $n=15,46.9 \%$ ), such as the development of a process map or task analysis $37,40,45,46,49-51,53,55,56,63,65-68$; statistical analysis $(n=11$, $34.4 \%)^{37,39,41,44,46,49,54,61,63,64,66}$; and two studies (6.3\%) conducted computer simulations. ${ }^{40,53}$ Supplementary File 3 provides full details of the analytical approaches. Table 2 presents the analytical approaches adopted according to the studies' use of a framework, theory or model, and whether the study explicitly referred to HFE within the manuscript. As represented in Table 2, studies which referred explicitly to HFE within the manuscript were more likely to have adopted qualitative analytical techniques. Studies which did not refer to HFE within the manuscript nor used or developed a framework, theory or model were more likely to apply statistical analysis and/or system representation analysis 
Four different analysis methods and 17 types of data collection approaches were adopted by the studies (with the majority of studies applying more than one data collection approach). The Mixed Method Appraisal Tool (MMAT) allows for assessment of both qualitative, quantitative and mixed methods. ${ }^{69}$ It was not appropriate in this instance as approximately half of studies $(n=15)$ conducted system representation analysis (e.g. hierarchical task analysis, process mapping), yet none of the MMAT questions were applicable to this specific analytical approach. Therefore, formal quality assessment of the studies was not conducted.

\section{Study foci}

The content analysis of the aim/objective(s) of each study identified three distinct study foci, as presented in Table 3. Over half of the studies ( $n=18,56.3 \%)$ explored aspects that contribute to errors or safety; 11 (34.4\%) explored the working processes of the pharmacy dispensary (e.g. tasks, workflow); and three studies explored compliance with procedures ( $n=3,9.4 \%)$.

\section{[Table 3. Overview of the Human Factors/Ergonomics (HFE) focus of included studies ( $n=32$ ) to be}

\section{displayed here]}

\section{Key findings}

The key findings will be presented as per the three study foci identified (Table 3)

Studies which explored aspects which contribute to errors or safety

The aspects perceived to contribute to errors or safety within the dispensing process related to: (i) the internal environment of the pharmacy setting, (ii) the usability and design of pharmacy technology, (iii) the dispensing task itself, (iv) organisational factors, (v) external influences, and (vi) patient-related contributors.

In relation to the internal environment of the pharmacy setting, perceived factors were interruptions and distractions ${ }^{39,} 42,46,48,58,60,61$; time pressures and workload $39,42,46,54,60,61,64$; the design of the dispensary ${ }^{42,46,48,62}$; staffing levels and skillset of pharmacy staff. ${ }^{46,54,60}$

The usability and design of tools and technology was commonly reported upon ${ }^{46,54,57-60,64}$ : automated dispensing technology and flawed computer software design were perceived to contribute to errors or safety, ${ }^{46,54,64}$ and prescription tracker technology was reported to have a negative effect on information flow. ${ }^{45}$ Four studies specifically explored the use of ePrescribing technology within community pharmacies. ${ }^{57-60}$ Safety concerns were identified in relation to the cognitive burden of staff secondary to memorising ePrescription information and performing mental calculations ${ }^{58}$; problematic design of the ePrescribing technology impacted workflow and potential harm ${ }^{57}$; textboxes could not accommodate the entirety of drug directions ${ }^{57}$; and there was inappropriate translation of 
information between prescribers and pharmacy staff. ${ }^{59}$ Harvey et al posed that the ability of pharmacy staff to successfully adopt ePrescribing technology depends if they are "technology oriented" pharmacies which rely on technology, or "socially oriented" pharmacies. ${ }^{43}$

In relation to the task of dispensing itself, a myriad of contributing factors were perceived to influence safety. Prescription factors influencing safety included illegible handwriting on prescriptions and complex prescriptions, ${ }^{46,48}$ and medications with similar names (i.e. look-alike and sound-alike drugs) ${ }^{39,41,48,61}$ and confusing names were considered to contribute to errors. ${ }^{61}$ 'Human error' of staff during dispensing included misreading the prescription ${ }^{39}$; automatically selecting a patient's previous drug or dose from their medication record ${ }^{39}$; forgetting to read carefully ${ }^{52}$; attention related issues ${ }^{52}$; and not checking the dose of a medicine prescribed. ${ }^{46}$

Organisationally within the dispensing setting, communication, teamwork and information exchange were reported as influential to safe and effective working, ${ }^{38,41,58,60,62}$ which themselves could be considered emergent outcomes of the system. Cooper et al identified that a fifth of error reports related to communication incidents, such as incomplete or delayed data transfer. ${ }^{41}$ Two studies identified that communication with both patients and other healthcare professionals were influential to safety. ${ }^{58,62}$

External influences posing risks to safety included lack of regulations and guidelines, ${ }^{38,55}$ and commercial pressures. ${ }^{38,62}$ Conflict was identified between the necessity of pharmacies to be profitable and the necessity for adequate resources (e.g. staffing) to maintain a safe working environment. ${ }^{62}$ The commercial nature of the community pharmacy setting was identified to increase local competition in one study, which resulted in the illegal supply of prescription medication. ${ }^{38}$ Patient-level contributors to safety were the presence of multi-morbidities ${ }^{41}$ and complex or agitated patients. ${ }^{46}$

\section{Suggestions posed in relation to safety concerns}

Suggestions were proposed as potential solutions to safety concerns. Commonly, this related to improving the design and usability of technology or software. ${ }^{46,52,57,59}$ Conversely, a number of studies recommended the adoption of technology to overcome safety concerns, including shared eHealth records, ${ }^{39}$ electronic prescribing technology, ${ }^{41}$ bar code scanning technology, ${ }^{41}$ prescription tracking technology, ${ }^{41}$ and automated dispensing technology. ${ }^{46}$ At pharmacy staff level, recommendations included continuous education and training ${ }^{39}$, 60; paying attention to staffing needs and communication skills ${ }^{39,60}$; better support and guidance regarding decision making ${ }^{41}$; employing a receptionist to answer the telephone ${ }^{46}$; and improved workforce planning. ${ }^{46}$ At a policy level, one 
study suggested increasing the number of inspectors to ensure safe practices ${ }^{39}$; another suggested a national policy on patient safety and incident reporting ${ }^{48}$; and the participants of one study advocated a maximum safe dispensing load. ${ }^{61}$ Phipps et al suggested the necessity of pharmacy protocols to be reviewed in terms of their relevancy, ${ }^{62}$ and Harvey et al suggested that core standards be set which can be dynamically adapted to changing environments (termed 'standard dynamic environments'). ${ }^{42}$

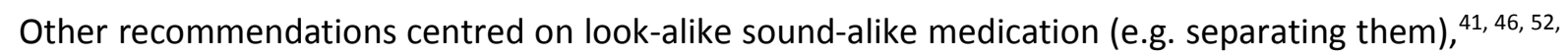
${ }^{61}$ improving the layout or design of the dispensing settings, ${ }^{42,46,61,64}$ sharing or reporting incidents, ${ }^{46}$, 61,64 and raising patient awareness of medication safety. ${ }^{39,52}$

\section{Studies which explored the working processes of the pharmacy}

Studies which explored the working process of the pharmacy can be subcategorised into those which: (i) explored the integration of a new technology or service, (ii) explored pre-existing dispensing processes, or (iii) focused on suggesting improvements to the dispensing process.

Two studies explored the implementation of technology or a new service. ${ }^{66,67}$ Successful integration of a patient education service within the dispensary workflow using process mapping was reported, ${ }^{67}$ and changes in workflow sequences following implementation of robotic dispensing technology increased efficiency and complexity. ${ }^{66}$

In relation to the exploration of pre-existing dispensing process, five studies focused on this. ${ }^{50,51,56,63,}$ ${ }^{68}$ Two studies focused on multi-tasking and task-switching, where it was identified that a pharmacist's work is highly mobile with high rates of task switching and multi-tasking, with interruptions and distractions considered the cultural norm. ${ }^{50,51}$ Naybour et al identified that different checking strategies (e.g. double checks) influenced the reliability and efficiency of the dispensing process ${ }^{56}$; Werner et al revealed a disconnect between a hospital satellite pharmacy, nursing units, and the overnight pharmacy ${ }^{68}$; and Phipps et al linked dispensing functions (e.g. clinical and medicine check) to high level pharmacy purposes (e.g. provision of medication and business viability). ${ }^{63}$

Four studies explored the pharmacy dispensing process with the purpose of suggesting improvements. $^{37,40,49,53}$ Two of these studies simulated the suggested changes within computer programs. ${ }^{40,53}$ Simulated process re-engineering and redistribution of medicine stock within a pharmacy increased the average number of prescriptions completed and decreased "slack time", ${ }^{40}$ and simulated changes to a pharmacy's design and work system improved the utilisation of staff time yet increased patient waiting times. ${ }^{53}$ Two studies did not simulate or implement suggestions posed but instead reported that the suggestions were used to support change. ${ }^{37,49}$ Aguilar et al suggested layout changes prevent interruptions and "traffic" within the pharmacy and to facilitate medication 
review and patient counselling, which were provided to management. ${ }^{37}$ Kumar and Kwong suggested changes to streamline the process of a pharmacy, which were reported to have initiated discussions. ${ }^{49}$

Each of the three studies which explored compliance to procedures identified that workarounds were a common occurrence. ${ }^{44,47,65}$ In relation to ePrescribing, Vassilakopoulou et al identified that the technology was not always used as intended or by authorised personnel, which was mostly due to organisational factors and the necessity of flexible working practices. ${ }^{65}$ Jones et al identified that pharmacy staff digress from procedures to maintain efficiency, which staff considered unlikely to adversely affect safety. ${ }^{47}$ Thus, Jones et al recommended that procedures and guidelines be made flexible, or a set of key procedures be identified to focus on. ${ }^{47}$ At the point of medication supply, Hoxsie et al identified that the name and date of birth of patients were not always verified, and differences in the compliance to procedures were observed between high risk and low risk pharmacies. ${ }^{44}$ Consequently, suggestions made included reviewing procedures, training staff on procedures, and having "secret shoppers" monitor compliance. ${ }^{44}$

\section{Recommendations for future research posed by the studies}

Twenty-three studies posed recommendations for future research. The recommendations of five studies centred on the necessity for further research, such as conducting larger scale studies. ${ }^{47,54,57 \text {, }}$ Three studies suggested further exploration of models developed within the study. This related to Mandt et al's model of "fast dispensing" and "active dispensing" 55 ; Jahn and Caldwell's CHIPPER framework ${ }^{45}$; and Harvey et al's sociotechnical model, which stratifies pharmacies into those which are "technically oriented", "socially oriented" or "improvising". ${ }^{43}$ Other recommendations were context-specific with little identifiable commonality between them. These recommendations related either to exploring potential solutions to safety concerns identified by the studies (such as proposed technological interventions), ${ }^{41,54}$ or recommendations for further exploratory research. ${ }^{38,39,52,53,55,58-}$ $60,62,64$

\section{Discussion}

This literature review identified a heterogeneous mix of studies adopting a HFE approach to explore the pharmacy dispensing process. Three distinct study foci were identified: aspects contributing to errors and safety within the dispensing process; the working processes of the dispensary; and compliance to dispensing procedures. Overall, four key findings emanated from this literature review. Firstly, factors influencing safety within the dispensing process range from the internal environment of the dispensary setting to external factors. Secondly, the dispensing process is complex in nature 
and can be depicted using a variety of system mapping approaches. Thirdly, deviations from procedures may be commonplace in pharmacy practice. And lastly, a plethora of methods, models, frameworks and theories exist through which HFE studies can be conducted.

297 The results demonstrate the variety of aspects influencing safety within the dispensing setting, ranging from the internal dispensing environment to external influences. The areas influencing safety identified are consistent with HFE theory, which poses that sociotechnical work systems function through the interaction of people, tasks, tools, technology, organisational context, and the internal/external environment. ${ }^{70}$ Alongside the multiple sources of risk identified within this review, it has been previously acknowledged that resultant dispensing errors can vary in nature ${ }^{23}$, and occur at various points throughout the dispensing process (e.g. transcription, validation, label production, preparation, selection, and delivery) as identified in the review by Stojkovic et al (2017). ${ }^{22}$ Considering this, it can be concluded that within the dispensing process there are multiple sources of risk, multiple types of errors, and various stages where errors can occur. This demonstrates the inherent complexity of safety within the dispensing process and corroborates that HFE is a promising platform through which the dispensing setting can be explored.

However, the state of current HFE research within the pharmacy setting is limited in two ways. Firstly, this review identified limited evidence that adopting HFE approaches within the pharmacy dispensing settings translates into improvements in safety and/or efficiency. Many studies made suggestions of changes which could improve safety or efficiency, yet none of these recommendations were reported to have been implemented, and only two studies simulated recommended changes within computer programs. ${ }^{40,53}$ This is consistent with the findings of Carayon et al on HFE research within patient safety. ${ }^{13}$ This review reported that few studies provided empirical evidence that HFE approaches improve patient safety, and when they do it is often limited to small-scale settings. Therefore, future HFE studies should provide evidence of the impact of a HFE approach on safety and/or efficiency to support the institutionalisation of HFE-based research within pharmacy practice. Secondly, few studies explored the pharmacy dispensing setting following a change in practice, such as the implementation of a new technology or service. This is despite previous suggestions that within the patient safety arena, HFE is well suited to explore the working environment following such changes in practice. ${ }^{13}$ Considering the advancement of the pharmacy profession and the necessity of appropriate work conditions to continue during organisational change to ensure staff engagement, ${ }^{71}$ future HFE studies within the pharmacy setting should explore changes in practice both prospectively and retrospectively to ensure the maintenance of safe working conditions. 
The findings also highlight known misconceptions surrounding healthcare technology. Some studies suggested technological innovations as solutions to overcome safety. However, it was conversely reported by other studies that flawed technological design negatively contributed to safety, and there is limited evidence that technological solutions within the dispensing process reduces risk. ${ }^{22}$ This suggests a mismatch between the perceptions of the influence of technology on safety in pharmacy, and the reality of its operation in routine practice in relation to errors. The belief that healthcare technology is easy to use and risk free is a known fallacy, ${ }^{72}$ and within the UK's National Health Service a poor fit between healthcare work systems and the design of technology is acknowledged. ${ }^{73}$ Aside from the usability of technology, additional requirements for successful implementation of healthcare technology include staff support for change and adequate resources. ${ }^{74}$ Based on the contradictory findings and a general paucity of research, further studies are needed to decipher how technology can be successfully implemented within the pharmacy dispensing setting in a way which does not negatively impact safety.

Workarounds were reportedly commonplace, indicating a potential misalignment between 'work as imagined' as stipulated within pharmacy procedures and the reality of 'work as done' within everyday practice. ${ }^{75}$ Within the wider domain of HFE research, workarounds are well acknowledged and are not necessarily perceived negatively, ${ }^{76}$ usually occurring in response to changing work environments where the rate of change is such that adaptation must occur..$^{77}$ Conversely, pharmacy staff have felt unsupported by employers in instances where they deviated from procedures, and have reported a tension between complying with pharmacy procedures and exercising their professional autonomy. ${ }^{78}$ This dichotomy was also evident in the current review; whilst one study appeared to champion the enforcement of procedures (e.g. through the use of secret shoppers), ${ }^{44}$ others advocated flexible procedures or 'standard dynamic environments' to allow for adaptation to occur appropriately. ${ }^{42,47,65}$ This is supported by Hollnagel et al who argue that within complex environments it is the flexibility of staff - and not compliance to procedures - which positively contributes to system performance. ${ }^{75}$ Considering these contrasting views and that workarounds were explored by only a small number of studies within this review, further exploration of workarounds and their impact within the dispensing process would be a compelling area for future research.

\section{The methodology of studies}

Many studies did not explicitly refer to a "human factors", "socio-technical", or "ergonomics" approach within the manuscript, despite each study's focus being within the wider domain of HFE. As the majority of studies were published in pharmacy or healthcare focused journals, the avoidance of this terminology may have been purposeful and instead language more amenable to the target audience used. Some of the studies' use of the terminology 'human error' can be considered 
problematic within the field of HFE as it suggests individual blame without considering the complex socio-technical environment contributing to this. Overall, this may indicate that the application and awareness of HFE research within pharmacy practice research is still within its infancy.

Only a minority of studies applied a framework, theory or model during the study design. When they were applied, a total of nine different ones were utilised, with only the Sociotechnical systems (STS) theory applied by more than one study. ${ }^{57,58}$ Whilst this indicates an extensive array of models, frameworks and theories which can be applied, the relative advantage of each is unknown and the most appropriate to apply in certain situations is unclear. The lack of a unified approach limits the comparison between studies and ultimately HFE understanding within the pharmacy dispensing arena. Considering the increasing drive to apply HFE approaches and systems thinking within healthcare, ${ }^{13}$ clear guidance on how to apply the different models, frameworks or theories may support future HFE research and eventual development of sound theory.

372

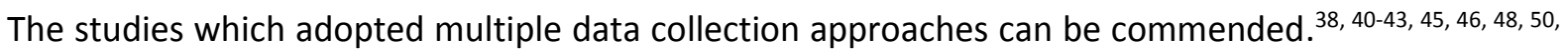
51, 54, 56-60, 63, 65-68 This considered a key feature of high-quality HFE research, as multiple data sources can facilitate the assessment of numerous facets of healthcare work systems, which ultimately leads to a better understanding of the system. ${ }^{79}$ It is also promising that qualitative methods were most commonly applied by the studies $38,41-43,46,47,50-52,55,57-60,62,65,67$ considering the viewpoint of Valdez and colleagues (2017) that qualitative research is vital to advance HFE and systems thinking in healthcare.$^{80}$ Interestingly, the studies which explicitly referred to HFE within the manuscript were more likely to have applied qualitative analysis, $38,43,47,52,57,58,60$ which may indicate the researchers had a sound knowledge of HFE and how to conduct such studies appropriately. Notably, the studies which did not explicitly refer to a HFE approach or apply/develop a framework, model or theory were

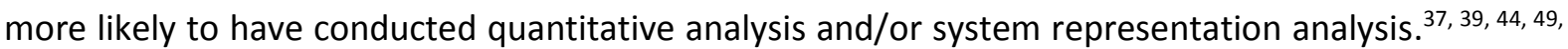
54, 61, 66 This may indicate that for these studies the researchers may not have been cognisant of the whole system throughout the study design.

\section{Recommendations for future research}

There was a lack of commonality in the future research suggested by the included studies. However, considering the uncertainty regarding the safety of technology within the dispensing setting, it may be valuable for future research to focus on the implementation of technological advancements within pharmacy practice. A timely example of such advancements is the European Union's (EU) Falsified Medicines Directive to be implemented in 2019, which necessitates all EU pharmacies to adopt barcode scanning technology to prevent the supply of falsified medicines to the public. ${ }^{81}$ Considering the scale of this adoption and the risk new technology poses on safe and effective pharmacy practice, 
exploring barcode scanning technology from a HFE perspective would be important in developing key recommendations to ensure its usability in practice and successful integration within processes.

\section{Strengths and limitations}

To structure the reporting of the results, the study findings were presented as per the three study foci identified. It should be acknowledged that each study focus may not exist in isolation and there may be interchangeable boundaries. An alternative approach could have been to conduct a qualitative synthesis of each study's results using a HFE framework which employs a whole systems perspective, such as the Systems Engineering Initiative for Patient Safety (SEIPS) model. ${ }^{70}$ However, this was purposefully not conducted as a more objective presentation of study results was considered more conducive to providing an overview of the state of HFE research conducted within the pharmacy dispensing setting.

A strength of this review is its inclusivity: studies were included if they applied a methodology or philosophy derived from HFE, even if it was not explicitly stated that an HFE approach was applied. This broadened the scope of eligible studies and prevented the exclusion of relevant studies; however, this selection process was subjective in nature and may have benefitted from a formalised checklist. All study types from any country were eligible for inclusion in this review, no time limits were applied to the search strategy, and studies from seven different countries were subsequently identified from a time period of 1995-2018. Additionally, studies conducted in both community pharmacy and hospital pharmacy dispensing settings were included. However, it should be noted that only five studies identified were conducted within hospital dispensary settings. Stojkovic et al (2017) only identified one study within the hospital dispensary setting, ${ }^{22}$ which alongside the findings of this review may indicate that this is an under-researched context. Therefore it is not currently possible to identify if there are unique differences between the community and hospital pharmacy dispensing setting. As identified within this review, the scope of research within the field of HFE is wide reaching and a heterogeneous mix of studies was identified. Considering the wide scope of the HFE discipline, it is possible that the search terms applied may not have identified all relevant studies. To circumvent this, a supplementary snowball search was conducted which involved screening the reference lists of identified studies and other publications by the authors. Due to the heterogeneity of the methods used in each study, formal quality assessment was not conducted as a quality assessment tool appropriate for each data collection method and analytical approach was not identified. The use of multiple quality assessment tools was unlikely to yield meaningful results. 
425 This review of the international literature identified that HFE studies within pharmacy dispensing settings have thus far focused on factors contributing to errors and safety, the working processes, and compliance to dispensing procedures. The complexity of the dispensing process has been clearly articulated which indicates the appropriateness of considering the adoption of a HFE approach when exploring this context to ensure researchers are cognisant of the whole system. Future HFE studies should focus on providing evidence of the impact adopting a HFE approach has on safety and/or efficiency, which in general would support its application in healthcare. Exploring changes in pharmacy practice using an HFE approach may ensure the continuation of safe working conditions, which may be of considerable value when implementing healthcare technology. Guidance on how the various HFE models, frameworks or theories can be helpfully applied would support such research and facilitate eventual development of theory.

\section{References}

1. Hignett $\mathrm{S}$, Jones $\mathrm{EL}$, Miller $\mathrm{D}$, et al. Human factors and ergonomics and quality improvement

2. Inernational Ergonomic Association. Definition and Domains of Ergonomics. https://www.iea.cc/whats/index.html; 2019 Accessed Jan 2019.

3. Karwowski W. Ergonomics and human factors: the paradigms for science, engineering, design, technology and management of human-compatible systems. Ergonomics. 2005;48:436-463.

4. Wilson JR. Fundamentals of ergonomics in theory and practice. Appl Ergon. 2000;31:557567.

5. Wilson JR. Fundamentals of systems ergonomics/human factors. Appl Ergon. 2014;45:5-13.

6. The World Health Organization. What is human factors and why is it important to patient safety? https://www.who.int/patientsafety/education/curriculum/who_mc_topic-2.pdf; 2019 Accessed March 2019.

7. Shorrock S, Williams C. Human Factors and Ergonomics in Practice: Improving System Performance and Human Well-Being in the Real World: CRC Press; 2016:4-5.

8. Jaffar N, Abdul-Tharim AH, Mohd-Kamar IF, Lop NS. A literature review of ergonomics risk factors in construction industry. Procedia Eng. 2011;20:89-97.

9. Salas E, Maurino D, Curtis M. Chapter 1 - Human Factors in Aviation: An Overview. In: Salas E, Maurino D, eds. Human Factors in Aviation (Second Edition). San Diego: Academic Press; 2010:3-19.

10. Parasuraman R, Riley V. Humans and Automation: Use, Misuse, Disuse, Abuse. Hum Factors. 1997;39:230-253.

11. Hignett $S$, Carayon $P$, Buckle $P$, Catchpole K. State of science: human factors and ergonomics in healthcare. Ergonomics. 2013;56:1491-1503.

12. Carayon $P, X i e A$, Kianfar $S$. Human factors and ergonomics as a patient safety practice. $B M J$ Qual Saf. 2014;23:196.

13. Carayon $\mathrm{P}, \mathrm{Xie} A$, Kianfar $\mathrm{S}$. Human factors and ergonomics as a patient safety practice. $B M J$ Qual Saf. 2014;23:196-205.

14. Joseph AC, Olivier S-C. From Discovery to Design: The Evolution of Human Factors in Healthcare. Healthc Q. 2012;15:24-29.

15. Reason J. Understanding adverse events: human factors. Qual Health Care. 1995;4:80-89. 
16. Bowie P, MacWalter G, Watson P, Gillies A, Pikcup L. A Scoping Review of Human Factors and Ergonomics Issues, Interventions and Resources in Primary Health Care 2017. Available from: https://learn.nes.nhs.scot/1601/patient-safety-zone/human-factors/scoping-reviewof-human-factors-and-ergonomics-issues; 2017 Accessed July 2019.

17. Department of Health Human Factors Reference Group. Interim Report, https://www.england.nhs.uk/wp-content/uploads/2013/11/DH-rep.pdf; 2012 Accessed April 2019.

18. U.S. Department of Veterans Affairs. VA National Center for Patient Safety: Human Factors Engineering. https://www.patientsafety.va.gov/media/hfe.asp; 2015 Accessed July 2019.

19. U.S. Department of Health and Human Services. Applying Human Factors and Usability Engineering to Medical Devices: Guidance for Industry and Food and Drug Administration Staff. https://www.fda.gov/media/80481/download; 2016 Accessed July 2019.

20. Al Juffali L, Al-Aqeel S, Knapp P, Mearns K, Family H, Watson M. Using the Human Factors Framework to understand the origins of medication safety problems in community pharmacy in Saudi Arabia: A qualitative study. Res Social Adm Pharm. 2018;15:558-567.

21. The World Health Organization. Essential Medicines and Health Products Information Portal: Medical Errors. http://apps.who.int/medicinedocs/en/d/Jwhozip02e/4.html; 2000 Accessed July 2019.

22. Stojkovic T, Marinkovic V, Manser T. Using prospective risk analysis tools to improve safety in pharmacy settings: A systematic review and critical appraisal. J Patient Saf. 2017.

23. James KL, Barlow D, McArtney R, Hiom S, Roberts D, Whittlesea C. Incidence, type and causes of dispensing errors: A review of the literature. Int J Pharm Pract. 2009;17:9-30.

24. Francis $M$, Hinchliffe $A$. Public Health Wales. Vaccination services through community pharmacy: a literature review. http://www.wales.nhs.uk/sitesplus/888/news/17071; 2010 Accessed July 2019.

25. Brown TJ, Todd A, Malley C, et al. Community pharmacy-delivered interventions for public health priorities: a systematic review of interventions for alcohol reduction, smoking cessation and weight management, including meta-analysis for smoking cessation. $B M J$ Open. 2016;6.

26. Lee $E$, Braund $R$, Tordoff J. Examining the first year of Medicines Use Review services provided by pharmacists in New Zealand: 2008. N Z Med J. 2009;122:3566.

27. Hatah E, Braund R, Tordoff J, Duffull SB. A systematic review and meta-analysis of pharmacist-led fee-for-services medication review. Br J Clin Pharmacol. 2014;77:102-115.

28. Emmerton L, Marriott J, Bessell T, Nissen L, Dean L. Pharmacists and prescribing rights: review of international developments. J Pharm Pharm Sci. 2005;8:217-225.

29. Cross VJ, Parker JT, Law Min M-CYL, Bourne RS. Pharmacist prescribing in critical care: an evaluation of the introduction of pharmacist prescribing in a single large UK teaching hospital. Eur J Hosp Pharm Sci Pract. 2018;25:e2-e6.

30. Frost TP, Adams AJ. Tech-Check-Tech in Community Pharmacy Practice Settings. J Pharm Technol. 2016;33:47-52.

31. Spinks J, Jackson J, Kirkpatrick CM, Wheeler AJ. Disruptive innovation in community pharmacy - Impact of automation on the pharmacist workforce. Res Social Adm Pharm. 2017;13:394-397.

32. Vosper $\mathrm{H}$, Hignett S. A UK Perspective on Human Factors and Patient Safety Education in Pharmacy Curricula. Am J Pharm Educ. 2017;82:6184.

33. Embrey MA. Ensuring Good Dispensing Practices. Managing Access to Medicines and Health Technologies: Kumarian Press; 2013:30.35.

34. Noyes J, Lewin S. Chapter 6: Supplemental Guidance on Selecting a Method of Qualitative Evidence Synthesis, and Integrating Qualitative Evidence with Cochrane Intervention Reviews. In: Noyes J, Booth A, Hannes K, et al., eds. Supplementary Guidance for Inclusion of 
Qualitative Research in Cochrane Systematic Reviews of Interventions. Version 1 (updated August 2011). 2011.

35. Popay J, Roberts H, Sowden A, et al. Guidance on the Conduct of Narrative Synthesis in Systematic Reviews. A Product from the ESRC Methods Programme. https://www.lancaster.ac.uk/shm/research/nssr/research/dissemination/publications/NS_S ynthesis_Guidance_v1.pdf; 2006 Accessed Jan 2019.

36. Dixon-Woods M, Agarwal S, Jones D, Young B, Sutton A. Synthesising qualitative and quantitative evidence: a review of possible methods. J Health Serv Res Policy. 2005;10:45-53.

37. Aguilar C, Chau C, Giridharan N, Huh Y, Cooley J, Warholak TL. How to plan workflow changes: a practical quality improvement tool used in an outpatient hospital pharmacy. $J$ Pharm Pract. 2013;26:214-219.

38. Al Juffali L, Al-Aqeel S, Knapp P, Mearns K, Family H, Watson M. Using the Human Factors Framework to understand the origins of medication safety problems in community pharmacy in Saudi Arabia: A qualitative study. Res Social Adm Pharm. 2018;15:558-567.

39. Ashcroft DM, Quinlan P, Blenkinsopp A. Prospective study of the incidence, nature and causes of dispensing errors in community pharmacies. Pharmacoepidemiol Drug Saf. 2005;14:327-332.

40. Chou YC, Chen BY, Tang YY, et al. Prescription-filling process reengineering of an outpatient pharmacy. J Med Syst. 2012;36:893-902.

41. Cooper A, Edwards A, Williams $\mathrm{H}$, et al. Sources of unsafe primary care for older adults: a mixed-methods analysis of patient safety incident reports. Age Ageing. 2017;46:833-839.

42. Harvey J, Avery AJ, Ashcroft D, Boyd M, Phipps DL, Barber N. Exploring safety systems for dispensing in community pharmacies: focusing on how staff relate to organizational components. Res Social Adm Pharm. 2015;11:216-227.

43. Harvey J, Avery AJ, Waring J, Barber N. The socio-technical organisation of community pharmacies as a factor in the Electronic Prescription Service Release Two implementation: a qualitative study. BMC Health Serv Res. 2012;12:471.

44. Hoxsie DM, Keller AE, Armstrong EP. Analysis of Community Pharmacy Workflow Processes in Preventing Dispensing Errors. J Pharm Pract. 2006;19:124-130.

45. Jahn MA, Caldwell BS. Community health integration through pharmacy process and ergonomics redesign (CHIPPER). Ergonomics. 2018;61:69-81.

46. James KL, Barlow D, Hiom S, Roberts D, Whittlesea C. Development and use of the critical incident technique in evaluating causes of dispensing incidents. Int J Pharm Pract. 2008;16:239-249.

47. Jones CEL, Phipps DL, Ashcroft DM. Understanding procedural violations using Safety-I and Safety-II: The case of community pharmacies. Saf Sci. 2018;105:114-120.

48. Koffuor GA, Anto BP, Abaitey AK. Error-provoking conditions in the medication use process: the case of a government hospital in Ghana. J Patient Saf. 2012;8:22-25.

49. Kumar S, Kwong AM. Six sigma tools in integrating internal operations of a retail pharmacy: a case study. Technol Health Care. 2011;19:115-133.

50. Lea VM, Corlett SA, Rodgers RM. Describing interruptions, multi-tasking and task-switching in community pharmacy: a qualitative study in England. Int J Clin Pharm. 2015;37:1086-1094.

51. Lester CA, Chui MA. Using link analysis to explore the impact of the physical environment on pharmacist tasks. Res Social Adm Pharm. 2016;12:627-632.

52. Lester CA, Kessler JM, Modisett T, Chui MA. A text mining analysis of medication quality related event reports from community pharmacies. Res Social Adm Pharm. 2018.

53. Lin AC, Jang R, Sedani D, Thomas S, Barker KN, Flynn EA. Re-engineering a pharmacy work system and layout to facilitate patient counseling. Am J Health Syst Pharm. 1996;53:15581564. 
54. Malone DC, Abarca J, Skrepnek GH, et al. Pharmacist workload and pharmacy characteristics associated with the dispensing of potentially clinically important drug-drug interactions. Med Care. 2007;45:456-462.

55. Mandt I, Horn AM, Ekedahl A, Granas AG. Community pharmacists' prescription intervention practices-Exploring variations in practice in Norwegian pharmacies. Res Social Adm Pharm. 2010;6:6-17.

56. Naybour M, Remenyte-Prescott R, Boyd M. Reliability Modelling of Dispensing Processes in Community Pharmacy. 10th IMA International Conference on Modelling in Industrial Maintenance and Reliability.2018.

57. Odukoya $\mathrm{O}$, Chui MA. Retail pharmacy staff perceptions of design strengths and weaknesses of electronic prescribing. J Am Med Inform Assoc. 2012;19:1059-1065.

58. Odukoya OK, Chui MA. e-Prescribing: characterisation of patient safety hazards in community pharmacies using a sociotechnical systems approach. BMJ Qual Saf. 2013;22:816-825.

59. Odukoya OK, Stone JA, Chui MA. E-prescribing errors in community pharmacies: exploring consequences and contributing factors. Int J Med Inf. 2014;83:427-437.

60. Odukoya OK, Stone JA, Chui MA. Barriers and facilitators to recovering from e-prescribing errors in community pharmacies. J Am Pharm Assoc (2003). 2015;55:52-58.

61. Peterson GM, Wu MS, Bergin JK. Pharmacist's attitudes towards dispensing errors: their causes and prevention. J Clin Pharm Ther. 1999;24:57-71.

62. Phipps DL, Noyce PR, Parker D, Ashcroft DM. Medication safety in community pharmacy: a qualitative study of the sociotechnical context. BMC Health Serv Res. 2009;9:158.

63. Phipps DL, Tam WV, Ashcroft DM. Integrating Data From the UK National Reporting and Learning System With Work Domain Analysis to Understand Patient Safety Incidents in Community Pharmacy. J Patient Saf. 2017;13:6-13.

64. Szeinbach S, Seoane-Vazquez E, Parekh A, Herderick M. Dispensing errors in community pharmacy: perceived influence of sociotechnical factors. Int J Qual Health Care. 2007;19:203209.

65. Vassilakopoulou P, Tsagkas V, Marmaras N. Workaround identification as an instrument for work analysis and design: a case study on ePrescription. Work. 2012;41 Suppl 1:1805-1810.

66. Walsh KE, Chui MA, Kieser MA, Williams SM, Sutter SL, Sutter JG. Exploring the impact of an automated prescription-filling device on community pharmacy technician workflow. J Am Pharm Assoc (2003). 2003;51:613-618.

67. Weir NM, Newham R, Corcoran ED, et al. Application of process mapping to understand integration of high risk medicine care bundles within community pharmacy practice. Res Social Adm Pharm. 2018;14:944-950.

68. Werner NE, Nelson ET, Boehm-Davis DA. Human factors methods to reduce medication error: using task analysis in a pediatric and adult pharmacy. Work. 2012;41 Suppl 1:56655667.

69. Hong QN PP, Fàbregues S, Bartlett G, Boardman F, Cargo M, Dagenais P, Gagnon M-P, Griffiths F, Nicolau B, O'Cathain A, Rousseau M-C, Vedel I. Mixed Methods Appraisal Tool (MMAT), version 2018. Registration of Copyright (\#1148552), Canadian Intellectual Property Office, Industry Canada.

70. Holden RJ, Carayon P, Gurses AP, et al. SEIPS 2.0: a human factors framework for studying and improving the work of healthcare professionals and patients. Ergonomics. 2013;56:1669-1686.

71. Dellve L, Strömgren M, Williamsson A, Holden RJ, Eriksson A. Health care clinicians' engagement in organizational redesign of care processes: The importance of work and organizational conditions. Appl Ergon. 2018;68:249-257.

72. Karsh B-T, Weinger MB, Abbott PA, Wears RL. Health information technology: fallacies and sober realities. J Am Med Inform Assoc. 2010;17:617-623. 
73. Waterson P. Health information technology and sociotechnical systems: A progress report on recent developments within the UK National Health Service (NHS). Appl Ergon. 2014;45:150-161.

74. Spetz J, Burgess JF, Phibbs CS. What determines successful implementation of inpatient information technology systems? Am J Manag Care. 2012;18:157-162.

75. Hollnagel E, Wears RL, Braithwaite J. From Safety-I to Safety-II: A White Paper. The Resilient Health Care Net: Published simultaneously by the University of Southern Denmark, University of Florida, USA, and Macquarie University, Australia,2015.

76. Hollnagel PE, Braithwaite PJ, Wears PRL. Resilient Health Care: Ashgate Publishing Limited; 2013.

77. Perry SJ, Wears RL. Underground adaptations: case studies from health care. Cogn Technol Work. 2012;14:253-260.

78. Thomas CEL, Phipps DL, Ashcroft DM. When procedures meet practice in community pharmacies: qualitative insights from pharmacists and pharmacy support staff. BMJ open. 2016;6:e010851-e010851.

79. Carayon P, Kianfar S, Li Y, Xie A, Alyousef B, Wooldridge A. A systematic review of mixed methods research on human factors and ergonomics in health care. Appl Ergon. 2015;51:291-321.

80. Valdez RS, McGuire KM, Rivera AJ. Qualitative ergonomics/human factors research in health care: Current state and future directions. Appl Ergon. 2017;62:43-71.

81. Royal Pharmaceutical Society. Falsified Medicines Directive. https://www.rpharms.com/resources/ultimate-guides-and-hubs/fmd; 2018 Accessed Jan 2019. 